\title{
TTR
}

Traduction, terminologie, rédaction

\section{Les mémoires de traduction et le rapport au texte : ce qu'en disent les traducteurs professionnels}

\section{Matthieu LeBlanc}

Volume 27, numéro 2, 2e semestre 2014

Traduction, textes, médias

Translation, Texts, Media

URI : https://id.erudit.org/iderudit/1037748ar

DOI : https://doi.org/10.7202/1037748ar

Aller au sommaire du numéro

Éditeur(s)

Association canadienne de traductologie

ISSN

0835-8443 (imprimé)

1708-2188 (numérique)

Découvrir la revue

Citer cet article

LeBlanc, M. (2014). Les mémoires de traduction et le rapport au texte : ce qu'en disent les traducteurs professionnels. TTR, 27(2), 123-148.

https://doi.org/10.7202/1037748ar

\section{Résumé de l'article}

Cet article présente les résultats partiels d'une étude ethnographique menée auprès de traducteurs professionnels. Dans son ensemble, l'étude porte sur l'utilisation des technologies langagières, notamment les mémoires de traduction, et la satisfaction du traducteur professionnel dans un environnement de travail de plus en plus " technologisé ». Pour les besoins de l'article, nous nous sommes limité à un aspect de l'étude, soit le rapport qu'entretiennent les traducteurs avec leur texte. Par exemple, de quelle manière le recours massif aux mémoires de traduction transforme-t-il le rapport au texte ? Le recours systématique aux mémoires de traduction est-il problématique à certains égards, comme l'avancent certains chercheurs ? Les traducteurs ont-ils l'impression de traduire de façon non linéaire, de se trouver devant un texte discontinu ? Le cas échéant, de quelle manière voient-ils ces changements au processus de traduction ? Pour répondre à ces questions, nous examinerons les résultats d'une étude réalisée dans trois entreprises et services de traduction situés au Canada, et qui nous a permis de mener des entretiens semi-dirigés auprès de plus de cinquante langagiers et d'observer les traducteurs à l'oeuvre. 


\title{
Les mémoires de traduction et le rapport au texte: ce qu'en disent les traducteurs professionnels
}

\author{
Matthieu LeBlanc \\ Université de Moncton
}

\section{Résumé}

Cet article présente les résultats partiels d'une étude ethnographique menée auprès de traducteurs professionnels. Dans son ensemble, l'étude porte sur l'utilisation des technologies langagières, notamment les mémoires de traduction, et la satisfaction du traducteur professionnel dans un environnement de travail de plus en plus «technologisé». Pour les besoins de l'article, nous nous sommes limité à un aspect de l'étude, soit le rapport qu'entretiennent les traducteurs avec leur texte. Par exemple, de quelle manière le recours massif aux mémoires de traduction transforme-t-il le rapport au texte? Le recours systématique aux mémoires de traduction estil problématique à certains égards, comme l'avancent certains chercheurs? Les traducteurs ont-ils l'impression de traduire de façon non linéaire, de se trouver devant un texte discontinu? Le cas échéant, de quelle manière voient-ils ces changements au processus de traduction? Pour répondre à ces questions, nous examinerons les résultats d'une étude réalisée dans trois entreprises et services de traduction situés au Canada, et qui nous a permis de mener des entretiens semi-dirigés auprès de plus de cinquante langagiers et d'observer les traducteurs à l'ouvre.

Mots-clés: traduction professionnelle, outils d'aide à la traduction, mémoires de traduction, segmentation du texte, approche ethnographique

\section{Abstract}

This article presents the partial results of an ethnographic study of professional translators. The study as a whole focuses on the use of language technologies, more specifically translation memory systems (TMs), and on the professional satisfaction of the translator in an increasingly technologized work environment. For the purposes of the article, we will focus on one aspect of our study: translators' relationship with their text. For example, how does heavy reliance on TMs change translators' relationship with their text? Is the systematic use of TMs problematic in some respects, as some researchers have posited? Do translators sometimes 
feel as if they are translating in a non-linear, discontinuous fashion? If so, how do they perceive those changes to the translation process? To answer these questions, we will examine the findings of our study conducted in three different translation firms and services located in Canada, and we will draw on data gathered in the workplace, including semi-directed interviews with over 50 language professionals and participant observation of translators at work.

Keywords: professional translation, computer-assisted translation tools, translation memories, text segmentation, ethnographic approach

\section{Introduction}

Look, now, at what happens when we use workstations that integrate translation memories and machine translation. The first thing you find is that the text is segmented, broken into units that sit one on top of the other. That is, the text is broken into paradigmatic form; its linearity is repeatedly interrupted. The translating mind is thereby invited to work on one segment after the other, checking for terminological and phraseological consistency but not so easily checking, within this environment, for syntagmatic cohesion. (Pym, 2011, p. 3)

Voilà ce qu'affirmait Pym dans un article sur l'influence des technologies langagières sur la traduction professionnelle. Ce qui ressort de cette affirmation, c'est l'effet de certains outils sur le texte dans son ensemble, plus précisément sur la segmentation du texte de départ et la non-linéarité du processus. C'est là, selon Pym, un changement radical par rapport à ce qui se pratiquait il y a dix ou quinze ans. Et c'est ce qui nous amène à nous interroger sur les changements qui sont survenus depuis l'intégration de certains outils d'aide à la traduction dans les milieux professionnels de la traduction. Vu l'ubiquité de ces outils, nous sommes d'avis qu'il s'agit là d'une question qui interpelle la traductologie.

Dans cet article, nous présenterons les résultats partiels d'une étude ethnographique qui porte sur les technologies langagières et le statut du traducteur. Dans cette étude, nous cherchons plus précisément à voir si, dans certains cas précis, le recours systématique à certains outils d'aide à la traduction, en particulier les mémoires de traduction, peut avoir une incidence sur le travail du traducteur à son sens large, sur sa satisfaction professionnelle et, partant, sur son statut professionnel. Pour ce faire, nous 
avons mené une étude sur le terrain, en milieu de travail, dans le but d'interroger les traducteurs et de les voir à l'œuvre. Nous nous limiterons, pour les besoins précis de cet article, au rapport qu'entretiennent les traducteurs avec leur texte, sachant fort bien que, depuis déjà plusieurs années, on discute abondamment des transformations engendrées en ce sens par le recours massif à certains outils d'aide à la traduction tels que les mémoires de traduction. Par exemple, le recours à ces outils est-il problématique, comme l'avancent certains? Le traducteur se trouve-t-il devant un texte discontinu, non linéaire? Traduit-il toujours segment par segment? Quel rapport entretient-il avec son texte? Et, surtout, qu'a-t-il à dire à ce sujet?

Seront donc présentés dans les sections qui suivent les résultats partiels de notre étude ${ }^{1}$. Après une brève mise en contexte et une présentation de l'état de la question, nous fournirons des précisions sur les milieux de travail à l'étude et sur l'utilisation des technologies par les traducteurs. Nous aborderons ensuite la question centrale de l'article, à savoir le rapport qu'entretient le traducteur avec son texte ${ }^{2}$, pour ensuite exposer les étapes suivantes de notre analyse et les pistes à suivre.

\section{Mise en contexte}

L'étude qui fait l'objet de cet article a des origines qui remontent à 2007, au moment où nous entamions, en collaboration avec une collègue, une recherche sociolinguistique qui nous a amené

1. Compte tenu de la récurrence des commentaires sur le «rapport au texte», nous avons jugé bon d'approfondir ce thème et d'y consacrer un article en entier; les autres aspects seront examinés ultérieurement (voir la conclusion pour des précisions supplémentaires).

2. Il est à noter que nous avons publié en 2013 un article qui porte sur l'étude ethnographique décrite dans le présent article («Translators on Translation Memory (TM). Results of an ethnographic study in three translation agencies and services", The International Journal of Translation and Interpreting Research. 5, 2, p.1-13). Dans l'article précité, qui donne une vue d'ensemble de la recherche, nous avons insisté sur les avantages et les inconvénients des mémoires de traduction aux yeux des traducteurs, que nous résumons dans la section 4.1. Dans le présent article, nous nous concentrons sur le rapport qu'entretiennent les traducteurs avec le texte de départ en insistant sur problèmes posés par la compartimentation/ segmentation du texte de départ (4.2.1), les manipulations supplémentaires exigées par la mémoire (4.2.2) et les effets sur le texte d'arrivée (4.2.3). Les conclusions des deux articles soulèvent ainsi des points différents. 
dans une importante entreprise canadienne de traduction ${ }^{3}$. Cette recherche exploratoire, qui, il importe de le préciser, ne portait pas expressément sur les technologies langagières, nous a permis de prendre conscience de l'ampleur de certains changements survenus au cours des dix dernières années dans les milieux de la traduction professionnelle. Dans l'entreprise en question, non seulement les exigences en matière de productivité avaient augmenté par suite de l'introduction d'outils d'aide à la traduction, mais les tâches du traducteur avaient été considérablement réduites. Autrement dit, les traducteurs étaient maintenant limités à certaines tâches bien précises, différentes de celles qu'ils exerçaient auparavant, et les mémoires de traduction, bien qu'utiles à certains égards, prenaient beaucoup de place dans le processus. C'est alors que nous nous sommes dit que cet aspect du travail, soit l'interaction entre les traducteurs et les mémoires de traduction, méritait d'être examiné de manière plus approfondie et systématique, et c'est précisément ce qui nous a amené à nous intéresser aux aspects humains de cette interaction.

\section{2. État de la question}

\subsection{Les mémoires de traduction}

Les mémoires de traduction font partie de ce que L'Homme appelle les «outils de rappel automatique» (2008, p. 174), qui reposent sur la réutilisation ou le recyclage des unités linguistiques. Comme le précise L'Homme, le «type de réutilisation effectuée par les mémoires de traduction repose sur l'établissement de correspondances entre des phrases à traduire et des phrases stockées dans un répertoire» (ibid.). Règle générale, les mémoires de traduction comportent un aligneur, un logiciel de terminologie et un dépouilleur (ibid.). Dans la majorité des cas, l'unité de segmentation des mémoires de traduction est la phrase (ibid.; v. aussi Bowker, 2002 et Kenny, 2011).

Commercialisées depuis le début des années 1990, les mémoires de traduction se sont largement répandues dans les milieux de la traduction, à tel point que, aujourd'hui, il s'agit de l'outil d'aide à la traduction le plus répandu dans le milieu de la traduction professionnelle (Bowker et Barlow, 2008; Christensen et Schjoldager, 2010). Les avantages mis de l'avant par les fabricants de mémoires de traduction sont nombreux, mais on

3. Voir LeBlanc, Dubois et Malaborza (2007). 
insiste notamment sur trois aspects, à savoir l'accroissement de la productivité des traducteurs (par le recyclage des traductions), l'amélioration de la qualité du produit fini (grâce notamment à l'uniformité terminologique) et l'augmentation du chiffre d'affaires des entreprises.

\subsection{La recherche empirique sur les mémoires de traduction}

Les chercheurs s'intéressent aux divers aspects des mémoires de traduction depuis un certain temps déjà. Dans leur synthèse des principales études menées en ce sens au cours des quinze dernières années, Christensen et Schjoldager (2010) affirment qu'il existe tout de même relativement peu d'études empiriques dans ce domaine. À titre d'exemple, certains chercheurs se sont penchés sur les avantages et les inconvénients d'une approche qui allie traduction automatique et mémoires de traduction (Lange et Bennett, 2000), alors que d'autres se sont intéressés à la segmentation du texte (Dragsted, 2004; Dragsted, 2006; Colominas, 2008) ou aux domaines et aux types de textes qui se prêtent le mieux aux mémoires de traduction. S'ajoutent à cela des recherches qui portent plus précisément sur le processus cognitif et l'effort requis de la part des traducteurs qui se servent des mémoires de traduction (O'Brien, 2006 et 2008). Enfin, certains ont étudié l'image que les traducteurs se font des mémoires de traduction (Dillon et Fraser, 2006) ${ }^{4}$.

Cela dit, on constate que peu d'études sur les mémoires de traduction ont été menées en milieu de travail, auprès des traducteurs. À ce propos, Cronin a déjà déploré «the absence of in-depth investigation of non-literary translation which relates it to larger questions of culture, society and language» (2003, p. 2), sans compter Mossop, qui signalait «the dearth of observations of the workplace» (2006, p. 789). Dans la même veine, Christensen et Schjoldager affirment que, s'agissant de la recherche sur les mémoires de traduction, il reste encore beaucoup de travail à faire : «Little research has been carried out on how translators interact with TM and how TM systems affect the cognitive (internal) translation process, and very few studies of TM are empirical investigations » (2010, p. 1-2). De là notre intérêt pour la recherche en milieu de travail.

4. Cette liste n'est pas exhaustive. Voir Christensen et Schjoldager (2010) pour une liste complète des recherches empiriques sur les mémoires de traduction. 


\subsection{Le discours sur les mémoires de traduction}

S'il est vrai que les mémoires de traduction offrent aux traducteurs et aux entreprises un certain nombre d'avantages - on pense notamment à un accroissement de la productivité et de l'uniformité dans les textes dans certains cas, à l'élimination de tâches répétitives et ennuyeuses dans d'autres -, il reste qu'elles font l'objet de critiques depuis déjà un certain temps. Les principaux reproches s'articulent autour de leurs effets "néfastes", notamment leur incidence sur le processus de traduction, et des problèmes litigieux qu'elles soulèvent en ce qui a trait à la tarification (Bowker, 2002, p. 121-122). C'est plus précisément à la fin des années 1990 qu'ont été formulées les premières critiques à l'égard des mémoires de traduction. Si la majorité de leurs détracteurs s'entendaient pour dire qu'elles offraient certains avantages non négligeables (v. supra), en revanche ils s'inquiétaient de leur incidence sur l'activité intellectuelle des traducteurs, c'est-à-dire sur le processus cognitif, du fait que les mémoires changent, dans l'essence, la façon de travailler, d'aborder le texte. Le principal reproche, c'est que les mémoires obligent les traducteurs à traduire phrase par phrase, ce qui fait que le processus est morcelé, le traducteur s'attardant surtout aux phrases - parfois même à des bouts de phrases - plutôt qu'au texte dans son ensemble. Comme le fait remarquer Évelyne Ross,

[1]'utilisation des mémoires de traduction exerce une influence sur certains processus mentaux chez le traducteur. Premièrement, elles font principalement appel aux microstratégies [, ce qui pourrait] avoir un impact négatif sur la qualité et accroître le temps qu'il met pour mener à bien sa tâche. En second lieu, elles impliquent de nouvelles stratégies d'évaluation que le traducteur ne possède pas encore. (2000, p. 17)

Bédard abonde dans le même sens en précisant que le principal défaut des mémoires est justement quelles forcent à traduire une phrase par une autre (rarement par deux phrases ou par rien du tout), alors que le traducteur traduit non pas des phrases mais bien un texte, un message (2000, p. 44). Cette façon de procéder aurait selon lui une incidence sur le texte, sur la distribution de l'information, sur l'articulation du message (ibid.). Bédard va même jusqu'à dire que, dans de telles circonstances, le traducteur subit une déformation professionnelle qui «l'amène, 
devant un paragraphe, à voir non pas le déroulement d'une idée, mais une simple collection de phrases. Certains pourraient avancer qu'il désapprend ainsi (déformation professionnelle) son métier» (ibid., p. 45). Il ajoute que le recyclage abusif aura certes une incidence sur la qualité du produit fini: discontinuités et erreurs terminologiques, erreurs de déictique, sens de la phrase (phrases tirées de différents contextes, par exemple). Cette façon de faire, à son sens, pourrait à la longue être une source de démotivation pour le traducteur, qui risque de devenir un «traducteur de phrases» (ibid.). S'agissant des erreurs, Bowker avait effectivement fait observer, au terme d'une étude menée auprès d'étudiants en traduction, que les traducteurs, du moins les débutants, risquent de se fier aveuglément aux solutions qui leur sont proposées par la mémoire de traduction (2005, p. 19).

Dans un même ordre d'idées, Mossop (2006) fait allusion à l'accroissement des exigences de productivité que l'on observe dans les divers milieux de la traduction depuis les 25 dernières années, accroissement qui viserait entre autres à rendre la traduction davantage productive. Le recours aux mémoires de traduction serait un des moyens qui permettrait aux entreprises et aux services de traduction d'accroître la productivité de leurs traducteurs (v. aussi Gouadec, 2007). À cela viendraient s'ajouter d'autres pratiques telles que le morcellement des textes - rendu plus facile grâce aux mémoires de traduction - et l'accroissement de la division du travail entre traducteurs et réviseurs:

It is possible that these changes are being accompanied by two significant changes in the mental process of translation. First, when chunking is combined with division of labour and less time for quality control, the result may be that very often no one has an overview of the text as a semantic whole. Second, the advent of electronic archives of old translations, together with large corporate and government Intranets containing original TL documentation, appears to be leading to a phenomenon we might call collage translation. In collage translation, composing a translation on a blank screen is replaced by revision of old TL material from a variety of sources to make it match the source text, together with varying amounts of effort to smooth the joints between the various parts of the resulting collage. (Mossop, 2006, p. 790) 
Et Mossop d'ajouter:

This way of creating translations involves a very different mental process from the traditional one of composing a translation on a blank screen. Is it a result of computerization? I suggest that the best way to answer this question is to say that the collage method of producing translations, while certainly enabled by information technology, is being driven by business pressures. (ibid.)

Ce qui ressort de cette dernière citation, c'est le lien quétablit Mossop entre l'usage que l'on fait des mémoires de traduction - ou qui est imposé par les employeurs - et le rétrécissement des délais impartis aux traducteurs.

Pym et Biau Gil reprennent bon nombre des arguments précités - à savoir l'incidence des mémoires sur le processus de traduction, la cohésion des textes et la qualité globale du message - en insistant sur le fait que le recours démesuré aux mémoires de traduction risque non seulement de démotiver les traducteurs, mais aussi de les isoler davantage: "It also means that translators are sometimes far removed from the end client and the overall context of the texts they work on [...]. The resulting work can be quite isolating and dehumanizing» (2006, p. 8). A leur sens, il s'agit là d'un recul à la fois pour les traducteurs et la profession dans son ensemble:

The industrial applications of translation memory tools are based on the idea that translation is a word-replacement activity. On the other hand, translation theories since the 1980s have tended to see translators as communicators whose duties go beyond the replacement of source-text words; translators are employed to provide meaningful communication. Translation memory makes this difficult. Indeed, they move translators back to the equivalence paradigm of the 1960s. Worse, now that texts commonly comprise not only written words but also images, videos and layout (think of any website), translation requires a division of labor. Thanks to our tools, translators are invited to forget about the other elements configuring the text. This division of labor may not always create satisfying long-term employment. (ibid., p. 11)

Enfin, dans un article sur les technologies et la traduction, Garcia (2009), à l'instar de Pym, se montre très critique à l'égard 
de certaines pratiques traductionnelles qui ont suivi l'implantation des mémoires de traduction. Selon lui, les technologies, plus précisément les mémoires de traduction, malgré leurs avantages indéniables, auraient dans certains cas pour effet de restreindre le rôle du traducteur en ce qu'elles transforment en quelque sorte la traduction en un travail de transcodage: les traducteurs se concentrent non plus sur le texte dans son ensemble mais plutôt sur les segments, les phrases (p. 202). À cela vient s'ajouter, dans certains cas, la traduction automatique [MT-assisted TM] pour la traduction de segments qui n'ont pas été récupérés dans la mémoire de traduction. Dans un scénario tel que celui-là, le traducteur ne fait que réviser le produit fini, en assure la postédition. Garcia estime que, dans de telles conditions, les traducteurs professionnels risquent de se faire supplanter par des non-professionnels:

Soon, if not already, professional translators in the localization industry will no longer translate texts (like their literary counterparts) or segments (as in the TM heyday), but just post-edit machine output [...] The new model will continue improving its cost-effectiveness by reducing demands on those doing the post-editing, and this will almost certainly see the deputising of competent bilinguals in place of professional translators. The present cycle will then presumably close, as translation loses its professional status and returns to its millennial amateur paradigm. (ibid., p. 208; v. aussi Garcia, 2007)

Au dire de Garcia, toute l'industrie de la traduction risque d'être marginalisée par les progrès technologiques, ce qui pourrait avoir des répercussions sur le statut du traducteur professionnel (2009, p. 210). Il est vrai qu'il fait surtout référence à la localisation et à la traduction technique, mais l'on sait très bien que, de plus en plus, on a recours aux mémoires de traduction pour la traduction de textes de toute nature.

C'est ainsi que, comme le suggère Kenny, nous nous devons de nous pencher sur les aspects problématiques des mémoires de traduction, de voir si les technologies peuvent dans certains cas avoir une incidence sur les conditions de travail des traducteurs, sur leur rémunération et sur leur image professionnelle (2011, p. 471; v. aussi Kenny et Way, 2001, p. 17). Somme toute, les remarques et les critiques formulées plus haut soulèvent d'importantes questions, questions qui, toutefois, doivent faire l'objet d'études empiriques. 


\section{Précisions sur l'étude}

\subsection{Méthodologie}

De nature largement qualitative, notre étude s'inspire en grande partie des techniques de recherche privilégiées en ethnographie. Il s'agit d'une approche qui nous a permis de nous intégrer aux différents services et entreprises de traduction afin de mieux en comprendre le fonctionnement et, surtout, de voir, de manière concrète, les traducteurs à l'œuvre dans leur environnement naturel. À l'instar de Blanchet (2000), nous nous sommes inspiré du modèle du «sablier ", démarche qui propose d'aller du global (c'est-à-dire de l'observation participante) au particulier (entretiens semi-dirigés, collecte de données contextuelles) pour revenir à une synthèse globale (interprétation des résultats). L'observation participante suppose une participation, voire une immersion totale et complète du chercheur dans le milieu qu'il étudie, l'objectif étant de mieux en comprendre la complexité et les subtilités: «cette méthode permet de vivre la réalité des sujets observés et de pouvoir comprendre certains mécanismes difficilement décryptables pour quiconque demeure en situation d'extériorité» (Soulé, 2007, p. 128; v. aussi Mucchielli, 2009). Dans notre cas, nos observations en milieu de travail se sont échelonnées sur presque trois mois, soit de janvier à mars 2012. Nous avons eu l'occasion d'observer (observation directe) les traducteurs à leur poste de travail dans le but de mieux voir comment ils travaillent. S'ajoutent à cela les entretiens semidirigés que nous avons menés auprès de plus de 50 langagiers (et d'autres employés), lesquels nous ont permis de recueillir, pour chacun de participants, non seulement des données personnelles, mais aussi des renseignements sur les pratiques traductionnelles et l'interaction avec les outils ${ }^{5}$. Les entretiens semi-dirigés offrent l'avantage de cadrer la discussion, au moyen d'une grille d'entrevue, tout en laissant la libre parole aux participants (voir la grille en appendice). Ils ont aussi ceci de particulier qu'ils permettent, par

5. À noter que nous ne cherchons pas, comme l'ont déjà fait d'autres chercheurs (O'Brien, 2006 et 2008, par exemple), à mesurer chacune des étapes du processus de traduction au moyen de loculométrie ou encore de la saisie de frappe. Il existe en effet déjà des études de ce genre sur l'effet des mémoires sur le processus cognitif de la traduction. Par ailleurs, il nous aurait été impossible, pour diverses raisons (y compris la confidentialité des données), d'avoir recours à ces techniques dans les milieux à l'étude. Notre étude est donc complémentaire à ces études. 
l'information recueillie, de valider et de compléter ce qui se dégage de l'observation directe et des données contextuelles recueillies.

Grosso modo, nos observations en milieu de travail ont permis 1) de nous familiariser avec le fonctionnement du service ou de l'entreprise à l'étude (S'agit-il d'un service public ou d'une entreprise qui obéit à une logique commerciale, par exemple? La productivité est-elle importante?) ainsi que le déroulement des opérations (tâches affectées à chacun, cheminement du texte, utilisation des outils, etc.), 2) d'observer les traducteurs à l'œuvre dans leur environnement de travail naturel et, de manière plus précise, de mieux comprendre comment ils interagissent avec les outils d'aide à la traduction, notamment les mémoires de traduction, 3) d'interroger les traducteurs sur divers aspects de leur travail (interaction avec les outils, objectifs de production, rationalisation des tâches, évolution de la profession, satisfaction professionnelle, statut du traducteur, etc.), 4) de discuter avec les gestionnaires, les chargés de projets, les techniciens, etc., dans le but de bien connaître tous les aspects de l'organisation du travail.

Enfin, il est à noter que l'approche ethnographique est de plus en plus courante en traductologie, de nombreux chercheurs ayant constaté qu'elle se prête bien à l'analyse des pratiques traductionnelles dans divers contextes (Flynn, 2011, p. 116). À l'instar de Koskinen, qui s'est intéressée à la traduction au sein d'un service de traduction de la Communauté européenne, nous sommes d'avis que cette approche, de par l'intégration au milieu qu'elle préconise, permet au chercheur d'examiner de près non seulement les textes mais aussi les traducteurs: «[...] both text and people in their habitat» $(2008$, p. 6). Dans notre cas, elle nous a également permis d'observer l'interaction avec les outils. Et, comme l'ajoute Koskinen, «ethnography pays equal attention to the minute details of everyday experience and to the structures and forces that bear on them» (ibid.).

\subsection{Les entreprises et services de traduction à l'étude}

Pour les besoins de notre recherche, nous avons eu l'occasion de passer trois semaines dans chacun des trois services et entreprises de traduction à l'étude. Il s'agit plus précisément de services gouvernementaux et d'entreprises privées situées au Canada. De manière à protéger la confidentialité des données et l'anonymat des participants, et conformément à l'Énoncé de politique des 
trois Conseils en matière de recherche avec des êtres humains (TPSGC, 2005), nous ne pourrons révéler le nom des services et des entreprises en question. Ce que nous pouvons dire, toutefois, c'est qu'ils comptent tous au moins 35 employés à temps complet: traducteurs (débutants, intermédiaires et chevronnés), réviseurs, correcteurs d'épreuves, terminologues, gestionnaires, personnel administratif, techniciens en informatique et spécialistes de l'éditique.

Dans les trois milieux à l'étude, la traduction se fait, sauf exception, de l'anglais vers le français, et l'on traduit des textes pragmatiques, non littéraires. Tous les traducteurs sont titulaires d'un diplôme universitaire, le plus souvent en traduction, et plusieurs sont agréés par une association professionnelle. Enfin, les traducteurs sont appelés à traduire des textes généraux (y compris des textes administratifs), techniques et spécialisés dans un grand nombre de domaines et pour divers clients.

Voici quelques données factuelles sur les trois services et entreprises à l'étude. (À noter que les noms que nous avons donnés aux services et entreprises sont fictifs.)

\section{Traduco}

- Nombre d'employés: 55-60 (dont 28 traducteurs et réviseurs)

- Types de textes traduits: textes généraux, techniques et spécialisés

- Mémoire de traduction: intégrée progressivement depuis 2006

- Nombre d'entretiens : 17 entretiens semi-dirigés (traducteurs, réviseurs et gestionnaire) et discussions avec l'ensemble des traducteurs, réviseurs et gestionnaires. Durée moyenne des entrevues: entre 25 minutes et 1 heure +

- Observation directe: 7 séances d'observation des traducteurs au travail (demi-journée $x 7$ )

- Total: à peu près 100 heures sur place

\section{LingExport}

- Nombre d'employés : 22-25 employés (dont 14 traducteurs et réviseurs)

- Types de textes traduits: textes généraux, techniques et spécialisés 
- Mémoire de traduction: intégrée progressivement depuis 2008

- Nombre d'entretiens: 19 entretiens semi-dirigés (avec traducteurs, réviseurs et gestionnaire) et discussions avec l'ensemble des traducteurs, réviseurs et gestionnaires. Durée moyenne des entrevues: entre 25 minutes et 1 heure +

- Observation directe: 7 séances d'observation des traducteurs au travail (demi-journée $x 7$ )

- Total: à peu près 100 heures sur place

\section{Globolingua}

- Nombre d'employés: 75-80 employés (dont 36 traducteurs et réviseurs ${ }^{6}$ )

- Types de textes traduits: textes généraux, techniques et spécialisés

- Mémoire de traduction: intégrée en 2001

- Nombre d'entretiens: 16 entretiens semi-dirigés (avec traducteurs, réviseurs, gestionnaires) et discussions avec la quasi-totalité des traducteurs et réviseurs sur place. Durée moyenne des entrevues: entre 15 minutes et 1 heure +

- Observation directe: 7 séances d'observation des traducteurs au travail (demi-journée $x 7$ )

- Total: à peu près 100 heures sur place

Nous avons, au total, passé à peu près 300 heures en milieu de travail, réalisé une cinquantaine d'entretiens semi-dirigés et effectué une vingtaine de séances d'observation de traducteurs à l'œuvre.

\subsection{L'utilisation des technologies langagières}

Les milieux qui ont fait l'objet de notre étude sont tous des environnements de travail très «technologisés» en ce sens que, dans l'exercice de leurs fonctions, les traducteurs ont recours à une gamme d'outils d'aide à la traduction (bitextes, dictionnaires en ligne, correcticiels, logiciels de gestion terminologique, etc.). Les mémoires de traduction sont quant à elles complètement intégrées aux postes de travail. Dans deux des trois milieux, cette intégration est tout de même assez récente (2006 et 2008), ce qui

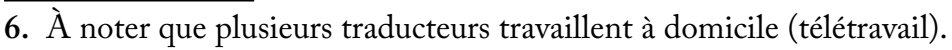


a permis aux traducteurs d'établir des comparaisons entre l'époque "pré-mémoire» et l'époque "post-mémoire». Ayant investi des sommes importantes dans les mémoires de traduction, les entreprises et services à l'étude tiennent à ce que leurs employés s'en servent afin de les alimenter, d'une part, et d'accroître la productivité et l'uniformité, d'autre part ${ }^{7}$. Dans les trois milieux, ce sont donc, à quelques exceptions près, tous les textes qui sont passés dans la mémoire de traduction ${ }^{8}$, peu importe la nature du texte, le destinataire, le sujet traité ou encore le nombre de textes en mémoire. Lobjectif est de constituer de nouvelles mémoires et d'alimenter les mémoires existantes.

\section{Résultats de l'analyse}

Découlent de l'analyse des données un certain nombre de constats sur tous les aspects du travail des traducteurs au sein des trois milieux de travail à l'étude: déroulement des opérations, cheminement des textes, outils d'aide à la traduction et interaction avec les divers outils, établissement des tarifs, contacts (ou non) avec les clients et avec les réviseurs, révision des textes, etc. Comme nous nous sommes intéressé surtout aux mémoires de traduction, nous avons cherché à voir de quelle manière les traducteurs se servent de cet outil et, surtout, à savoir ce qu'ils ont à dire à leur sujet (opinons, jugements). S'ajoutent à cela nos observations des traducteurs à l'œuvre, à leurs postes de travail, qui ont permis de compléter et de valider les données recueillies en entrevue (v. 4.2.4).

\subsection{Une vue d'ensemble: les "pour» et les «contre» des mémoires de traduction}

Même si nous nous concentrerons sur un seul des aspects qui touche aux mémoires de traduction, c'est-à-dire le rapport au texte, nous avons cru bon de présenter sommairement les principaux avantages et inconvénients des mémoires de traduction tels qu'ils ressortent de l'analyse'.

S'agissant des avantages, la majorité des traducteurs interrogés sont d'avis que les mémoires de traduction sont dans certains cas

7. Il s'agit de mémoires de traduction dites «classiques», dans lesquelles les textes sont divisés en segments.

8. Font parfois exception les textes très courts ou les textes de nature confidentielle.

9. Voir LeBlanc (2013) pour une liste détaillée des avantages et des inconvénients. 
d'un grand secours. Les avantages cités ont trait à l'accroissement de la productivité, à l'amélioration de l'uniformité des textes, à l'élimination du travail répétitif, à la convivialité des diverses fonctions intégrées aux mémoires de traduction (pour la recherche, par exemple) et à l'intérêt pédagogique qu'elles présentent (partage de solutions, par exemple). Les désavantages, pour leur part, sont tout aussi nombreux. Selon les traducteurs interrogés, les mémoires auraient pour effet de transformer le rapport au texte, de freiner la créativité, d'encourager la passivité, de nuire aux réflexes naturels, de créer une dépendance, de faciliter la propagation des erreurs et d'automatiser le travail. Nous n'entrerons pas, faute d'espace, dans les détails, mais il suffit de dire que les données recueillies sont riches et soulèvent de nombreuses questions qui ont trait non seulement au traducteur, mais également à ses conditions de travail et à son statut professionnel.

\subsection{Les traducteurs, les mémoires de traduction et le rapport au texte}

S'il est un commentaire qui revient dans la quasi-totalité des entretiens et qui ressort des discussions que nous avons eues avec les traducteurs et réviseurs, c'est celui du rapport quentretiennent les traducteurs avec le texte de départ et, dans une certaine mesure, avec le texte d'arrivée. La grande majorité des traducteurs s'entendent pour dire que l'un des principaux inconvénients de la mémoire de traduction, c'est la segmentation du texte de départ quelles entraînent (v. Dragsted, 2006). Par segmentation, on entend le «phrase par phrase» ou le «segment par segment», une manière de procéder qui est plus ou moins inévitable lorsqu'on se sert d'une mémoire de traduction (v. section 2.3). Trois sous-thèmes se dégagent de l'analyse, bien qu'ils soient tous étroitement liés et en fait inséparables: 1) la compartimentation/segmentation ${ }^{10}$ du texte de départ; 2) les manipulations (supplémentaires) exigées par la mémoire; 3) l'effet sur le texte d'arrivée (fluidité, incongruités, contexte).

\subsubsection{La compartimentation/segmentation du texte de départ}

Lorsqu'on aborde la question des mémoires de traduction, l'une des premières remarques - le plus souvent non sollicitée - est celle

10. Ce sont les termes segmentation et compartimentation qui ressortent le plus souvent dans les entretiens. 
de la compartimentation ou de la segmentation du texte de départ qu'elles entraînent. Il importe de rappeler que la majorité des traducteurs interrogés ont connu l'époque "pré-mémoire» et sont donc en mesure de se prononcer sur les changements qui ont suivi l'implantation des mémoires de traduction. Qu'en est-il donc?

Pour certains traducteurs, la façon de procéder, bien qu'elle soit solidement implantée, est tout ce qu'il y a de moins naturel en ce sens que, de par leur conception, les mémoires de traduction exigent que les traducteurs procèdent phrase par phrase. Voici ce que nous dit l'une des traductrices interrogées à ce sujet:

La mémoire m’a forcée à procéder phrase par phrase, alors que ce nétait pas du tout comme ça que je procédais [avant l'introduction des mémoires de traduction]. On voyait au moins le paragraphe [...]. Ça change beaucoup la façon d'aborder un texte. (TRI $2 \mathrm{~B}^{\mathrm{II}}$ )

Pour d'autres, c'est justement le fait de ne pas avoir une vue d'ensemble du texte de départ, d'avoir à travailler dans une boîte d'édition en mode interactif, qui constitue le principal irritant:

Les mémoires de traduction, ça entraîne une segmentation du texte à traduire qui fait qu'on a souvent tendance à oublier que la traduction n'est pas une activité segmentée. Quand on veut traduire une phrase de l'anglais [sans mémoire de traduction], on peut souder des éléments ensemble, mais la disposition du TD et des boîtes [d'édition] font en sorte qu'on a tendance à oublier cela: on travaille phrase par phrase. C'est cette idée de compartimentation. Certains des traducteurs que je révise ont maintenant tendance à oublier le contexte, à oublier ce qui vient avant et à ne pas tenir compte de ce qui vient après. C'est l'un des effets les plus pernicieux des mémoires. (TRI3A)

Cette dernière remarque est celle d'une traductrice-réviseure d'expérience qui constate depuis déjà plusieurs années les effets de la segmentation sur les textes traduits par les débutants qu'elle révise. Elle fait notamment référence au contexte et à la cohésion du texte d'arrivée. Une autre traductrice d'expérience abonde dans le même sens en insistant sur les limites du phrase par phrase: "On ne voit pas ce qui vient avant ou ce qui vient après. Parfois, on pourrait regrouper des phrases, mais on n'est pas portés à le

11. Code des participants. 
faire pour que ce soit plus idiomatique, [car] sous les yeux on n'a qu'une seule phrase à la fois » (TR I IC).

Interrogés sur ce qu'ils entendaient par l'absence d'une vue d'ensemble du texte de départ, les traducteurs ont confirmé qu'il était bien sûr possible d'avoir, à leurs côtés, une copie imprimée du texte ou d'en avoir une copie électronique, mais que cela exige un effort supplémentaire.

Enfin, les traducteurs débutants auxquels nous avons fait allusion plus haut sont souvent eux-mêmes conscients des limites de cette approche et des difficultés qu'elle entraîne. En témoignent les commentaires de cette jeune traductrice, qui constate les différences entre les méthodes et techniques préconisées à l'université et la façon dont elle aborde aujourd'hui ses textes au moyen de la mémoire de traduction:

Ça change, on ne peut pas le nier, ça change le procédé de traduction [...]. À l'université, on lit la phrase, on pense aux différentes façons, on pense au message; maintenant, je peux traduire la moitié de la phrase sans avoir lu le reste, justement en raison de la façon dont la mémoire de traduction fonctionne. (TRO5A)

Plus tard dans l'entretien, elle revient sur cette façon de faire, qu'elle dit déplorer malgré tout.

Dans l'ensemble, on s'aperçoit que la compartimentation/ segmentation du texte de départ demeure problématique pour la majorité des traducteurs interrogés, même si, par la force des choses, de nombreux traducteurs se sont habitués à cette façon de faire.

\subsubsection{Les manipulations (supplémentaires) exigées par la mémoire}

Dans un même ordre d'idées, ce que déplorent certains traducteurs, c'est que la mémoire de traduction influe sur d'autres parties du processus et exige d'eux un effort supplémentaire pour fusionner ou souder des phrases, alors que ce sont là, à leur dire, des opérations tout à fait normales et naturelles du processus de traduction. À cela s'ajoutent les déplacements d'éléments, qui s'avèrent toujours plus compliqués que par le passé, au dire des traducteurs. Voici ce que nous confie à ce sujet l'une des traductrices interrogées:

C'est bizarre de travailler une phrase à la fois. Souvent, à la fin d'un texte, je pense à un changement que je voudrais 
apporter au début, et il faut retrouver la case, fusionner des segments. Ce n'est pas une façon naturelle de traduire; il faut s'y habituer. (TRO5A)

Et d'ajouter une autre traductrice: «On revient au phrase par phrase, et si on regroupe, ça bousille tout» (TR I 2B). Ce que l'on observe, c'est qu'une opération autrefois courante et normale - à savoir le déplacement d'éléments à l'intérieur d'un texte ou encore la fusion de deux ou plusieurs phrases - constitue maintenant une opération plus complexe, qui ne cadre pas avec la logique interne des mémoires de traduction. Quelques-uns ont même avoué qu'ils hésitent à fusionner ou à scinder des phrases de crainte de nuire à l'alignement des textes dans la mémoire.

Parallèlement, les traducteurs se prononcent aussi sur les effets de cette segmentation sur le texte d'arrivée. De l'avis d'une des traductrices interrogées,

Côté contexte, c'est vraiment facile de s'éloigner du texte et complètement oublier ce qu'il y avait plus loin, de faire la liaison avec les phrases, d'ajouter des charnières pour que le texte soit plus lisible, c'est plus compliqué [...]. Pour avoir une vue d'ensemble du texte, il faut faire un effort. (TRO5C)

On voit qu'elle abonde dans le même sens que la traductrice citée en 4.2.1, qui affirmait qu' "[o]n ne voit pas ce qui vient avant ou ce qui vient après» (TRI IC). À leurs yeux, il ne fait aucun doute que les manipulations exigées par la mémoire pour fusionner ou souder des phrases constituent une source d'irritation en ce sens qu'elles sont contraignantes et vont à l'encontre du processus normal de traduction. Et d'aucuns estiment que cette façon de faire se répercute sur la qualité du texte d'arrivée.

\subsubsection{Effets sur le texte d'arrivée (fluidité, incongruités, contexte)}

Aux nombreux commentaires sur la segmentation du texte de départ viennent s'ajouter, comme nous venons de le voir, des remarques sur l'incidence des mémoires sur la qualité du produit fini. Pour certains, la compartimentation ne peut faire autrement que d'avoir un effet sur la cohérence et la cohésion du texte d'arrivée. Comme le fait observer cette traductrice,

Le gros inconvénient [des mémoires de traduction], c'est la fluidité du texte [d'arrivée]; c'est le fait de découper le 
texte en petits tronçons alors qu'en français on aurait relié deux phrases, on aurait donné plus de fluidité au texte d'arrivée; avec les mémoires, ce n'est pas vraiment possible de le faire. [...] Ça a cassé le rythme de certains textes. (TRI2A)

Cette même traductrice ajoute que «le segment peut constituer une barrière psychologique à une bonne traduction. Le danger, c'est que ça mécanise les choses, le danger de prendre un segment qui ressemble au sien mais qui n'a rien à voir avec son contexte $[\ldots]$ » (TR I 2A). Elle s'interroge en plus sur l'utilisation des mémoires de traduction par les traducteurs débutants:

[Les débutants] s'habituent à la mécanisation de la traduction et ne font plus l'effort de sortir de la mémoire. [...] On ne voit plus le texte qu'au travers des cases. [...] Ça met un filtre terrible [entre le traducteur et son texte] et la nouvelle génération [les traducteurs débutants] est dans un carcan dont elle n'a même pas conscience qu'elle peut se défaire. (TRI 2A)

Il est vrai que cette dernière remarque peut, à première vue, avoir l'air quelque peu alarmiste. Pourtant, la traductrice en question n'est pas du tout réfractaire aux technologies ou encore aux mémoires de traduction. Malgré les inconvénients qu'elle fait ressortir et les préoccupations qu'elle exprime, elle constate que les avantages des mémoires de traduction sont tout de même assez nombreux. Elle soulève toutefois des questions on ne peut plus importantes sur l'effet des mémoires de traduction à la fois sur le processus de traduction et sur la qualité du texte d'arrivée, à l'instar d'une autre traductrice: "Cette façon de faire [le phrase par phrase] donne lieu à toutes sortes d'incohérences [dans le texte d'arrivée] et l'on s'en rend compte à la lecture finale du texte» (TRoIC). Comme d'autres, cette traductrice est d'avis que les effets se font sentir en langue d'arrivée (qui est toujours le français dans le cas de cette étude), mais il faudrait bien sûr mener des études à grande échelle (et longitudinales) pour le vérifier.

Enfin, pour certains, les mémoires auraient un effet non seulement sur la qualité du texte d'arrivée, en raison notamment de la segmentation qu'elles imposent, mais également sur le processus de création: 
J'estime que la traduction, c'est un processus de création, et dès lors que la mémoire suggère quelque chose, c'est comme si le robinet se fermait chaque fois. C'est trop compartimenté, ça interrompt le flux de la création, et chaque fois que j'ai une phrase qui m'est proposée, je perds la fluidité de mes pensées et je prends ça pour du cash. Un gros zéro comme aide. [...] La phrase qui suit celle qui t'a été proposée par la mémoire te force à faire des gymnastiques qui ne sont peut-être pas naturelles, parce que, disons, la phrase commence par tel sujet et pour enchaîner il faut exactement le même sujet, alors que toi, tu aurais procédé autrement et ça te serait venu plus naturellement par la suite; c'est comme s'il y avait une interruption. [...] Cela va prendre plus de temps, car il faut que je refasse des choses qui me seraient venues plus naturellement si ça ne m'avait pas été proposé. (TRI 9B)

Pour cette traductrice-réviseure chevronnée, la segmentation du texte de départ aurait un réel effet sur le processus de création en ce sens que les solutions proposées par la mémoire viennent s'interposer entre le traducteur et son texte, commentaire qui rejoint celui d'un autre traducteur d'expérience: "C'est une approche plus froide qu'on a. Il y a une machine entre nous et le texte, qui nous impose quelque chose, qui brime notre liberté de traducteur» (TRO6B).

Dans l'ensemble, l'analyse révèle que bon nombre de traducteurs sont convaincus que la segmentation du texte de départ a des effets sur le produit fini, d'une part, et sur le processus de création, d'autre part.

\subsubsection{Lien avec les observations des traducteurs à l'œuvre}

Comme nous l'avons mentionné plus haut, notre étude comportait aussi plus de 20 séances d'observation de traducteurs à l'œuvre. Compte tenu du cadre restreint de cet article, il nous sera impossible de décrire par le menu détail toutes les conclusions que nous avons tirées de nos observations ${ }^{12}$, mais nous sommes néanmoins en mesure d'affirmer que les problèmes occasionnés par la segmentation du texte sont perceptibles dans bien des cas. D'abord, dans la quasi-totalité des cas, les traducteurs traduisent effectivement phrase par phrase (et parfois segment

12. Voir LeBlanc (2013). 
par segment), comme l'impose la mémoire de traduction, et cela, indépendamment de la nature du texte, de la proportion du «déjà traduit» (correspondances partielles ou parfaites) ou encore des délais impartis. C'est une façon de procéder qui semble bien ancrée dans les trois milieux. Chose intéressante, il est même des traducteurs qui, pendant les séances d'observation, ont attiré notre attention sur la question de la segmentation en faisant référence aux entretiens. Enfin, nous avons pu, à cinq reprises, témoigner de visu des problèmes que peut occasionner cette approche (erreurs de déictique; mauvaise interprétation du contexte; cohésion). Somme toute, nos observations nous ont permis de vérifier ce qui se dégageait des entretiens, et vice-versa.

\subsection{Quelques avantages de la compartimentation/ segmentation du texte}

S'agissant de la segmentation, il serait faux de croire que tous les traducteurs interrogés sont du même avis. En effet, pour une poignée de traducteurs, le mode de fonctionnement des mémoires de traduction a ceci d'avantageux qu'il permet au traducteur de mieux découper le texte, de focaliser toute son attention sur la phrase. D'où la remarque suivante d'une traductrice débutante: «La mémoire nous force à procéder phrase par phrase, mais cela nous permet de nous concentrer sur chaque phrase» (TRO5C). De l'avis de cette dernière, il y a, de cette façon, moins de risque d'omettre des éléments de sens qui doivent figurer dans le texte d'arrivée. Et une traductrice-réviseure d'ajouter: «[La mémoire] permet de mieux voir les découpages»(TRO6A). Toujours est-il que ce genre de commentaire se fait plutôt rare.

\section{Conclusion}

Il importe de rappeler que nous avons insisté, dans cet article, sur un seul aspect de notre recherche de terrain, à savoir le rapport qu'entretient le traducteur avec son texte. Notre analyse révèle effectivement que la question du rapport au texte, plus précisément la segmentation du texte et ses effets sur le processus et le produit fini, est un thème récurrent dans les entretiens et les discussions. Sur les 51 traducteurs interrogés, plus de la moitié ont abordé cette question en début d'entrevue, souvent sans que nous ayons à soulever la question. De par leur conception, les logiciels de mémoires de traduction, malgré leurs différences inhérentes, 
imposent une façon de travailler qui ne fait évidemment pas l'unanimité parmi les traducteurs interrogés. D'une part, cette façon de faire va à l'encontre des principes de base de la traduction tels qu'on leur a enseignés à l'université, et d'autre part, elle se distingue nettement de la façon dont ils traduisaient avant l'implantation des mémoires de traduction. Mais avant tout, la mécanisation de l'opération, le "phrase par phrase», fait en sorte que, le plus souvent, les traducteurs n'ont devant eux, à l'écran, que des segments, des phrases, ce qui donne une impression de nonlinéarité, de compartimentation qui, à leurs yeux, pose problème pour les raisons exposées plus haut. Dans une certaine mesure, les traducteurs interrogés confirment certaines des réserves et préoccupations émises par Ross (2000), Bédard (2000), Mossop (2006), Gouadec (2007), Pym et Biau Gil (2006) ainsi que Garcia (2009) sur l'effet des technologies sur le texte. Fort conscient que notre étude se limite à trois milieux de travail et qu'il nous est donc impossible de généraliser les résultats, il y a tout de même lieu de croire que, à s'en tenir aux similitudes que nous avons constatées entre les trois milieux, la situation décrite n'est très probablement pas un cas isolé.

Il reste maintenant à pousser plus loin l'analyse des données et à examiner de plus près les pratiques qui sont privilégiées par les entreprises et services qui ont fait l'objet de notre étude. Par exemple, dans quelle mesure les mémoires ont-elles un effet sur les exigences de productivité des traducteurs? Quel degré de liberté les traducteurs ont-ils par rapport au «déjà traduit», plus précisément les correspondances parfaites? Leurs tâches ont-elles été réduites au fil des années? Et y a-t-il un lien entre l'usage qu'on fait des technologies, les pratiques traductionnelles qui sont privilégiées, les conditions de travail et la satisfaction professionnelle des traducteurs? Voilà autant de questions que nous examinerons dans la suite de notre analyse. Mais tout compte fait, nous sommes d'accord avec Dillon et Fraser (2006) pour dire qu'il importe de mener davantage d'études qualitatives, d'interroger les traducteurs, de voir ce qu'ils ont à dire. Et comme le font observer Taravella et Villeneuve (2011), il faut se pencher sur les aspects humains des technologies langagières dans l'organisation - un aspect dont on a peut-être trop peu tenu compte jusqu'à maintenant - et, comme le suggèrent Dam et Zethsen (2009), sur le statut professionnel du traducteur. 


\section{Références}

Bédard, Claude (2000). «Mémoire de traduction cherche traducteur de phrases». Traduire, 186, p. 41-49.

Blanchet, Philippe (2000). La linguistique de terrain: méthode et théorie. Une approche ethno-sociolinguistique. Rennes, Presses universitaires de Rennes.

Bowker, Lynne (2002). Computer-Aided Translation Technology: A Practical Introduction. Ottawa, Presses de l'Université d'Ottawa.

Bowker, Lynne (2005). «Productivity vs. Quality? A Pilot Study on the Impact of Translation Memory Systems». Localization Focus, 4, 1, p. 13-20.

Bowker, Lynne et Michael Barlow (2008). «A Comparative Evaluation of Bilingual Concordancers and Translation Memory Systems». In E. Yuste Rodrigo, dir. Topics in Language Resources for Translation and Localisation. Amsterdam/Philadelphie, John Benjamins, p. 1-22.

Christensen, Tina Paulsen et Anne Schjoldager (2010). «TranslationMemory (TM) Research: What Do We Know and How Do We Know It?» Hermes - The Journal of Language and Communication, 44, p. 1-13.

Colominas, Carme (2008). «Towards Chunk-based Translation Memories». Babel, 54, 4, p. 343-354.

Cronin, Michael (2003). Translation and Globalization. Londres et New York, Routledge.

Dam, Helle V. et Karen Korning Zethsen (2009). «Who Said Low Status? A Study on Factors Affecting the Perception of Translator Status». JoSTrans - The Journal of Specialised Translation, 12, p. 2-36.

Dillon, Sarah et Janet Fraser (2006). "Translators and TM: An Investigation of Translators' Perceptions of Translation Memory Adoption». Machine Translation, 20, 2, p. 67-79.

Dragsted, Barbara (2004). Segmentation in Translation and Translation Memory Systems - An Empirical Investigation of Cognitive Segmentation and Effects of Integrating a TM System into the Translation Process. Copenhague, Samfundslitteratur.

Dragsted, Barbara (2006). «Computer-aided Translation as a Distributed Cognitive Task». Pragmatics E Cognition, 14, 2, p. 443-464.

Flynn, Peter (2011). «Ethnographic Approaches». In Y. Gambier et L. van Doorslaer, dir. Handbook of Translation Studies (vol. 1). Amsterdam/Philadelphie, John Benjamins, p. 116-119.

Garcia, Ignacio (2007). "Power Shifts in Web-based Translation Memory». Machine Translation, 21, p. 55-68.

Garcia, Ignacio (2009). «Beyond Translation Memory: Computers and 
the Professional Translator». JoSTrans - The Journal of Specialised Translation, 12, p. 199-214.

Gouadec, Daniel (2007). Translation as a Profession. Amsterdam/ Philadelphie, John Benjamins.

Kenny, Dorothy et Andy Way (2001). «Teaching Machine Translation and Translation Technology: a Contrastive Study». In M. L. Forcada et al., dir. MT Summit VIII - Proceedings of the Workshop on Teaching Machine Translation (Sept. 2001, Santiago de Compostela). Genève, EAMT.

Kenny, Dorothy (2011). «Electronic Tools and Resources for Translators». In K. Malmkjaer et K. Windle, dir. The Oxford Handbook of Translation Studies. Oxford, Oxford University Press, p. 455-472.

Koskinen, Kaisa (2008). Translating Institutions. An Ethnographic Study of EUTranslation. Manchester, St. Jerome

Lange, Carmen Andres et Winfield Scott Bennett (2000). «Combining Machine Translation with Translation Memory at Baan». In R. Sprung, dir. Translating into Success. Amsterdam/Philadelphie, John Benjamins, p. 203-218.

LeBlanc, Matthieu, Dubois, Lise et Sonya Malaborza (2007). «"Même en Acadie, on peut bien traduire": traduction et technologisation en milieu minoritaire». Communication présentée dans le cadre du colloque «Tradaptation, technologie et nomadisme» tenu à l'Université Concordia du 8 au 10 mars 2007.

LeBlanc, Matthieu (2013). «Translators on Translation Memory (TM). Results of an ethnographic study in three translation services and agencies». The International Journal of Translation and Interpreting Research, 5, 13, p. 1-13.

L'Homme, Marie-Claude (2008 [2000]). Initiation à la traductique. Montréal, Linguatech.

Mossop, Brian (2006). «Has Computerization Changed Translation?» Meta, 51, 4, p. 787-793.

Mucchielli, Alex, dir. (2009). Dictionnaire des méthodes qualitatives en sciences humaines ( $3^{\mathrm{e}}$ éd.), Paris, Armand Colin.

O'Brien, Sharon (2006). «Eye-tracking and Translation Memory Matches». Perspectives: Studies in Translatology, 14, 3, p. 185-205.

O'Brien, Sharon (2008). «Processing Fuzzy Matches in Translation Memory Tools: An Eye-tracking Analysis». Copenhagen Studies in Language, 36 («Looking at Eyes: Eye Tracking Studies of Reading and Translation Processing»), p. 79-102.

Pym, Anthony et José Ramón Biau Gil (2006). «Technology and Translation (Pedagogical Overview)». In A. Pym, A. Perekrestenko et B. Starink, dir. Translation Technology and Its Teaching. Tarragona, 
Universitat Rovira i Virgili et Intercultural Studies Group.

Pym, Anthony (2011). «What Technology Does to Translating». The International Journal for Translation and Interpreting Research, 3, 1, p. 1-9.

Ross, Évelyne (2000). «Usage des mémoires de traduction». Traduire, 186, p. 7-18.

Soulé, Batstien (2007) «Observation participante ou participation observante? Usages et justifications de la notion de la participation observante en sciences sociales». Recherches qualitatives, 27, 1, p. 127140.

Taravella, AnneMarie et Alain O. Villeneuve (2011). «Aspects humains des technologies langagières dans l'organisation». Communication prononcée dans le cadre de la conférence Tralogy, Paris, mars 2011.

Travaux publics et Services gouvernementaux Canada [TPSGC] (2005 [1998, 2000, 2002]. Énoncé de politique des trois Conseils: Éthique de la recherche avec des êtres humains, Ottawa, Secrétariat interagences en éthique de la recherche.

Matthieu LeBlanc

Département de traduction et des langues

Faculté des arts et des sciences sociales

Université de Moncton

Moncton (Nouveau-Brunswick) E1A 3E9

CANADA

matthieu.leblanc@umoncton.ca 


\section{Appendice}

\section{Grille d'entretiens}

\section{Renseignements personnels}

- Nom, prénom et âge

- Lieu d'origine et de résidence

- Scolarisation et formation

- Poste occupé

- Aperçu des tâches et responsabilités (hiérarchie)

- Répertoires linguistiques

- Rôle au sein de l'organisme

- Objectifs personnels poursuivis

- Satisfaction quant à l'expérience

Renseignements sur l'interaction du traducteur avec les outils de travail (traduction assistée par ordinateur)

- Liste des outils employés (survol des outils)

- Outils informatiques

- Outils «papier»

- Aisance à manipuler les outils d'aide à la traduction

- Logiciels de mémoire de traduction (MT)

- Connaissance du logiciel et aisance d'utilisation

- Différences: traduire avec la MT et sans la MT

- Avantages de la mémoire de traduction

- Inconvénients de la mémoire de traduction

- Mémoire de traduction et créativité

- Mémoire de traduction et liberté d'expression

- Mémoire de traduction et satisfaction personnelle

- Mémoire de traduction et «déqualification»

- Vitesse et productivité

- Satisfaction personnelle et professionnelle

- Statut du traducteur et avenir professionnel

\section{Autres questions pertinentes}

- Norme linguistique

- Formation professionnelle (préparation, pertinence)

- Avenir du français et de la traduction

- Le statut du traducteur 